\title{
Design of Compound Machine Tool for Ultra-Precision Shaft Parts
}

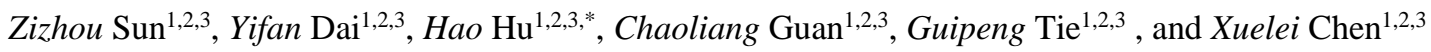 \\ ${ }^{1}$ College of Intelligent Science and Technology, National University of Defense Technology, Changsha 410073, China \\ ${ }^{2}$ Hunan Key Laboratory of Ultra-Precision Machining Technology, Changsha 410073, China \\ ${ }^{3}$ Laboratory of Science and Technology on Integrated Logistics Support, National University of Defense Technology, Changsha \\ 410073, China
}

\begin{abstract}
Ultra-precision shaft components are widely used, such as the shaft core of air-floating spindle, etc. At present, the final precision of such workpieces are difficult to reach through CNC machining tools, but often with the help of manual grinding, of which the machining precision and efficiency are greatly limited. Based on the deterministic figuring method, this paper uses an abrasive belt polishing machine to carry out ultra-precision figuring experiments on shaft parts. On this basis, an ultra-precision composite machine tool for shaft parts is designed with the functions of turning, grinding and abrasive belt polishing. The dynamic performance requirements of the machine tool are put forward by analyzing the precision index of the workpiece and the error control principle of deterministic figuring, and the structure of machine tool is designed and the performance parameters of each part are selected. combined with the finite element analysis, the key components are optimized to meet the machining requirements of $0.1 \mu \mathrm{m}$ roundness and $1 \mu \mathrm{m}$ cylindricity on the steel shaft.
\end{abstract}

\section{Introduction}

Ultra-precision shaft components are widely used in ultra-precision machining and measuring equipment, precision instruments, aerospace industry and other fields. Among them, as the equipment for manufacturing precision shafts, ultra-precision machine tools have higher requirements for the precision of their own airfloating spindles, which can reach $15-50 \mathrm{~nm}$ rotating accuracy[1]. For example, Moore's 500FG ultra-precision machine tool has a spindle rotating accuracy of $25 \mathrm{~nm}$; Taylor Hobson's cylindricity measuring instrument has a spindle rotating accuracy of $15 \mathrm{~nm}$. The main factors affecting the precision of the air-floating spindle rotation include the cylindricity of the shaft and the roundness of the shaft journal[2].

At present, high-precision shaft parts are usually processed by lathes and grinders. As the precision of traditional mechanical processing parts will not be higher than that of the machine tool's own components, if we want to further improve the precision, it will place too high requirements on the machining precision of the machine tool's own components, which can often only be achieved by manual polishing. Take a 1045 steel shaft with diameter of $100 \mathrm{~mm}$ and length of $150 \mathrm{~mm}$ as an example, after precision turning, the roundness is 1.5$2 \mu \mathrm{m}$, and the cylindricity is $5-10 \mu \mathrm{m}$, after ultra-precision cylindrical grinding, it can reach the roundness of 0.2$0.3 \mu \mathrm{m}$ and the cylindricity of $0.5-0.7 \mu \mathrm{m}$. After the final manual grinding of the air-floating spindle shaft core, the roundness of the shaft can reach $0.1 \mu \mathrm{m}$, and the cylindricity is about $1 \mu \mathrm{m}$. Manual grinding is nondeterministic processing, and the removal amount of each grinding is highly dependent on the experience of the technician, and the processing efficiency is low.

Many scholars have studied how to improve the precision of the shaft parts. Zhu and Jun applied fast tool servo technology to precision turning of shafts[3]. Aleksandrova used algorithms to optimize the machining parameters in the grinding process to improve their surface quality. Zhang and Yang used ELID precision mirror grinding technology to perform ultra-precision grinding experiments[4]. Chen introduced the idea of deterministic figuring of optical processing into the process of improving the precision of shaft parts and verified the feasibility of the theory and proved its strong potential[5]. However, due to the lack of dynamic performance and motion precision of current machine tools, the precision has reached a plateau.

In order to realize the digital figuring of ultraprecision shaft parts, based on the theory of deterministic figuring, this paper designs a device for deterministic figuring of ultra-precision shaft parts, the structural design and the performance parameters of each part were determined by the functional requirements, the principle of deterministic figuring, and the dynamic response of the dwell time solution requirements, finally realize the digital processing of ultra-precision shaft parts.

*Corresponding author: tiny_hh@139.com 


\section{Functional requirements for machine design}

We carried out deterministic figuring verification experiments on a principle-verification machine modified from a lathe. Five iteration experiments were performed on a 1045 steel shaft with a diameter of $100 \mathrm{~mm}$ after turning. The results are shown in Figure 1.



(a)

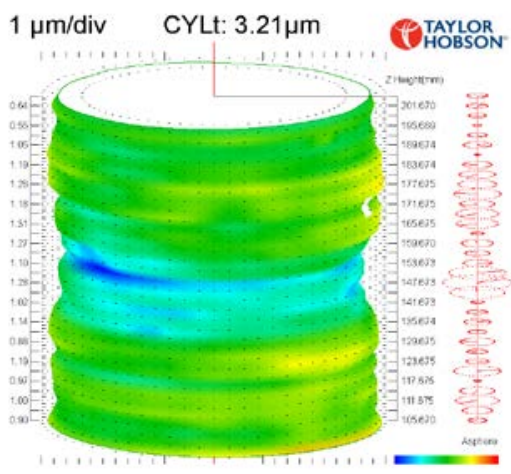

(b)



(c)

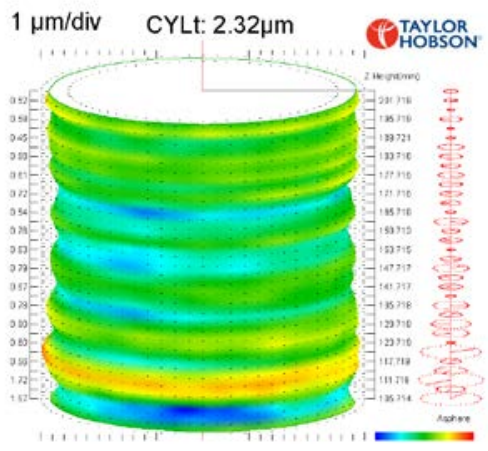

(d)

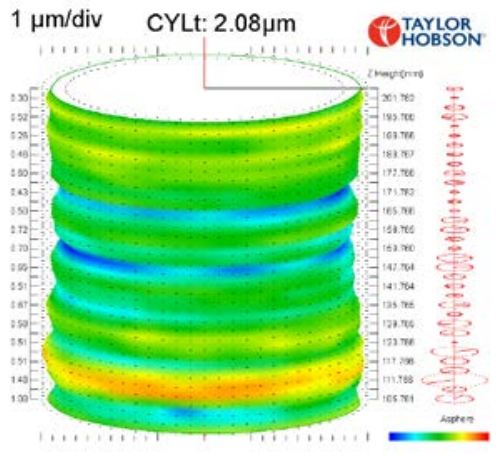

(e)

Fig. 1. The measurement results of shafts after 5 times of iterative machining. (a): After the first figuring; (b): After the second time; (c): After the third time; (d): After the fourth time; (e): After the fifth time.

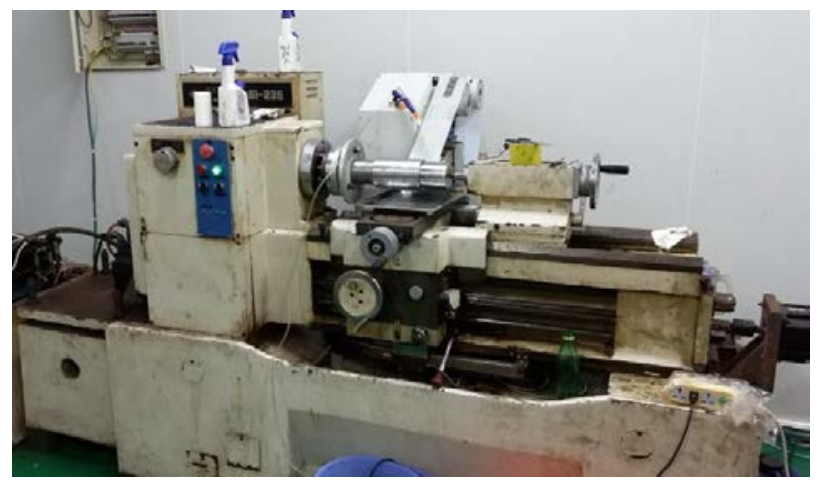

Fig. 2. The modified deterministic figuring machine used for principle verification.

On the principle-verification machine, which is shown in Figure 2, the roundness was converged from 1$1.2 \mu \mathrm{m}$ to $0.25-0.7 \mu \mathrm{m}$, and the cylindricity was converged from $6.8 \mu \mathrm{m}$ to $2.1 \mu \mathrm{m}$, and the cylindricity convergence ratio is 3.23, the result has reached the precision of precision cylindrical grinding machine. In addition, the experiment also verified the phenomenon that the convergence efficiency between two iterative experiments gradually decreases and the total convergence ratio is limited during the deterministic figuring process. Therefore, it is determined through software simulation and actual processing that the entrance conditions of the polishing process should reach $1 \mu \mathrm{m}$ roundness and $3 \mu \mathrm{m}$ cylindricity.

On this basis, in order to improve the equipment processing efficiency and improve the level of production automation, in addition to the ultra-precision polishing functions of the cylindrical surface, the machine should also have turning and grinding functions on the shaft's outer surface. The main processing objects of this machine tool are typical high-precision shaft parts with a diameter of $<150 \mathrm{~mm}$ and length of $<400 \mathrm{~mm}$, such as the air-floating spindle core of an ultra-precision machine. At the same time, it can also be used for the processing of some typical optical parts such as mirror blanks or lens tube. The entire machine tool should meet the CNC machining requirements of the above parts.

\section{Structural design and selection of components}




\subsection{Overall design of the machine tool}

According to the design function requirements, the machine tool uses a lathe as prototype, referring to the design structure of a mature ultra-precision drum lathe[6,7]. The machine is mainly composed of a spindle, a tailstock, a turning/grinding module, an abrasive belt polishing module, and a linear motion axis in $\mathrm{Z}$ and $\mathrm{X}$ directions. The overall structure is shown in Figure 3.



Fig. 3. Overall structure design drawing of the machine tool.

Among them, there are three linear motion axes in $\mathrm{Z}$ direction, one of which is for the tailstock to move independently, and the other two $\mathrm{Z}$ axes are each equipped with an $\mathrm{X}$ motion axis for installing the turning/grinding module and the abrasive belt polishing module. In order to meet the machining precision requirements in Section 2, the influence of factors such as high precision, high stiffness, temperature, and vibration must be considered in the structural design. The main design is around the bed design with high rigidity and good vibration absorption, and high precision servo feed system and other aspects.

\subsection{Design of key components in machine tools}

\subsubsection{Machine bed and base design}

The bed is processed from a whole piece of granite. Granite has the advantages of low linear expansion coefficient, good vibration resistance, high rigidity, no rust, and no magnetic response. It is widely used in various types of ultra-precision machine tools. Therefore, we choose granite as the basic bed material; At the same time, because cast iron has the characteristics of good vibration absorption and good workability, we choose cast iron as the support of the bed.

\subsubsection{Design and characteristics of abrasive belt polishing module}

The general structure of the polishing module is shown in the Figure 4. It mainly includes a vibrating contact wheel, a belt wheel, a reel wheel, and a cylinder ejection mechanism.



(a)

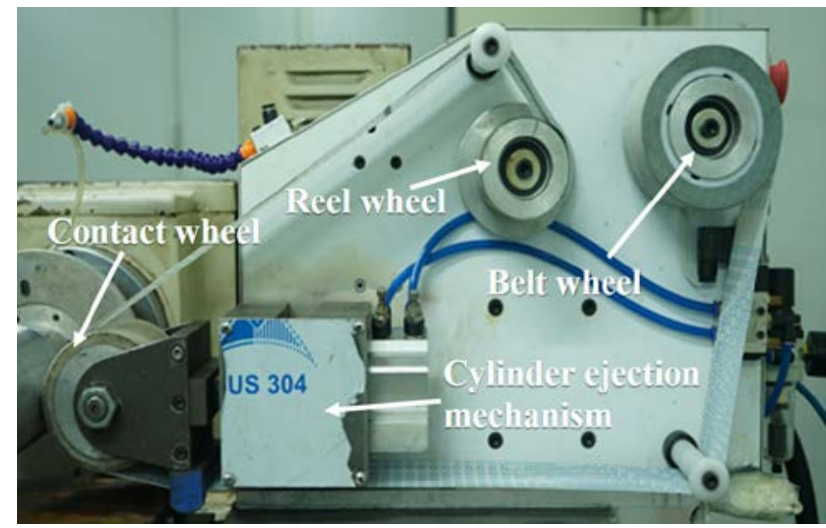

(b)

Fig. 4. Abrasive belt polishing mechanism. (a): Way of working; (b): Mechanism composition.

The contact wheel is ejected by the air cylinder to contact the workpiece at a constant pressure, and the material is removed by high-frequency vibration along the axis of the workpiece. The abrasive belt is updated by the rotation of the belt wheel and the reel wheel. The amount of deterministic figuring removal follows the Preston equation:

$$
\Delta H=K p v
$$

Where $\Delta H$ is the amount of material removed per unit time, $K$ is Preston constant, which is related to factors such as workpiece's material, abrasive and temperature. $v$ is the relative speed between the workpiece and the abrasive belt, and $p$ is the positive pressure between the workpiece and the contact wheel. According to the equation, as long as the contact pressure, the belt grinding speed, the abrasive belt and the material being processed are not changed, stable and accurate material removal can be achieved. Chen's experiment also verified the stability of the material removal method of abrasive belt polishing.

There are mainly three cutting parameters in traditional cutting processing: cutting depth $a_{p}$, feed speed $f$ and cutting speed $v$, and $a_{p}$ is the main factor affecting the improvement of the current traditional machine tool's machining precision. As an example of a cylindrical grinder, the straightness of $\mathrm{Z}$ axis guide, the rotation error of the grinding wheel spindle, and the coaxiality between spindle and tailstock will directly 
affect the $a_{p}$, which is reflected on the workpiece in the form of "error repetition".

Compared with traditional lathe and grinder, the main advantage of using deterministic polishing equipment is that deterministic figuring no longer requires $a_{p}$, but more easily controlled pressure $p$ and dwell time $t$. Because the abrasive belt always contacts the shaft surface with constant pressure, even if there is straightness error in $\mathrm{Z}$ axis and rotation error in spindle, constant pressure contact can offset these effects, therefore, the requirements for the precision of the machine tool are reduced. Compared with manual polishing, this equipment can greatly improve the processing efficiency, and the removal amount at each point can be more stable and accurate. This processing method is in line with the development direction of automation and intelligent equipment, and has great potential and broad application prospects.

\subsubsection{Linear motion axis design}

According to the polishing process entrence conditions given in Section 2, and considering the large cutting forces and vibrations generated during the turning and grinding process, we use a hydrostatic guide driven by linear motor on $\mathrm{Z}$ axis at which the turning/grinding module is installed, because it has a very high motion precision, good vibration resistance and large bearing capacity[8]. The other two $\mathrm{Z}$ motion axes and two $\mathrm{X}$ motion axes use linear guides driven by ball screw, and the models are selected according to the load. According to the calculation of the design index, the maximum stroke of $\mathrm{Z}$ axes is $500 \mathrm{~mm}$, and $250 \mathrm{~mm}$ on $\mathrm{X}$ axes.

Except for the hydrostatic guide, the other linear motion axes are driven by servo motors. Grating ruler with precision of $\pm 1 \mu \mathrm{m}$ can be mounted on each linear axis for full closed-loop position control except Z2 axis. $\mathrm{Z} 2$ axis uses motor encoder to achieve semi-closed-loop position control, while using motor current feedback to adjust the workpiece's clamping force.

According to the dwell time calculated by the figuring process software, the maximum speed can be obtained using Equation 2:

$$
v_{\max }=\frac{\mathrm{d} L}{\mathrm{~d} t}
$$

Where $d L$ is the discrete interval of the axial error data, which is set as $1 \mathrm{~mm}$ in program; $\mathrm{d} t$ is the dwell time. The minimum dwell time obtained according to the program is $1.33 \times 10^{-4} \mathrm{~min}$, the maximum speed of $\mathrm{Z3}$ axis should be not less than $125.3 \mathrm{~mm} / \mathrm{s}$ by calculation. According to the load and power, the screw diameter is chosen as $25 \mathrm{~mm}$ and the lead is $5 \mathrm{~mm}$, the rated speed of the servo motor is 3000RPM. So the actual maximum speed can reach $250 \mathrm{~mm} / \mathrm{s}$, which meets the requirements of the processing requirements.

\subsubsection{Selection of spindle and tailstock}

In the actual operation of this machine tool, vibration and impact will be generated against the spindle during turning and grinding, the weight of the steel shaft being processed requires a large bearing capacity of the spindle, and the speed of the shaft should exceed the maximum speed required by the figuring process. The hydrostatic spindle has good rotating accuracy and larger bearing capacity than the air-floating spindle, which make it widely used in heavy-load ultra-precision machine tools[9]. Define $\mathrm{d} L$ in Equation 2 as the discrete interval in the circumferential direction, which is set as $1^{\circ}$ in program, it can be calculated that the rotating speed of the $\mathrm{C}$ axis should be larger than 20.83RPM in polishing process. Besides, turning generally requires a speed of 500-600RPM. Therefore, selecting a hydrostatic spindle and tailstock with a rated speed of 600 RPM can meet the machining conditions.

\subsection{Precision of key components}

As stated in Section 3.2.2, turning and grinding processes are highly dependent on the accuracy of the machine parts. In order to make sure whether the parts after turning and grinding meet the entrance conditions of polishing process, we need to analyze the precision of key components[10]. For the processing of shaft parts, the geometric tolerances in the vertical plane is a nonsensitive direction of error, the effect on roundness and cylindricity is less than $10^{-4}$ orders of magnitude. So we mainly consider the geometric tolerances in the horizontal plane. The motion accuracy of key components is listed in Table1:

Table1. Precision of key components of machine tool.

\begin{tabular}{|c|c|}
\hline Items & Precision index \\
\hline Straightness of hydrostatic guide & $0.5 \mu \mathrm{m} / 200 \mathrm{~mm}$ \\
\hline Rotating accuracy of hydrostatic spindle & $<0.2 \mu \mathrm{m}$ \\
\hline
\end{tabular}

Because the base of the spindle and the tailstock is designed with an adjustable mechanism in the horizontal direction, by using a standard cylindrical rod and dial indicator, the parallelism in the horizontal plane between the machining reference axis and the hydrostatic guide can be adjusted to $1 \mu \mathrm{m}$. According to the data in Table1, it can be obtained that the theoretical cylindricity of a 200mm-long shaft after turning and grinding can reach $1.5 \mu \mathrm{m}$ and roundness can reach $0.67 \mu \mathrm{m}$. Both the roundness and the cylindricity can meet the entrance condition of the abrasive belt polishing process.

\section{FEM analysis and machining simulation}

\subsection{FEM deformation analysis}

For the structural design, we need to control the actual deformation of each part. We use FEA software to analyze and optimize the design based on the calculation results. Taking the granite bed as an example, it is the 
base on which all components are installed, and we need to control its deformation. We analyzed the bed supports using FEM software.

As can be seen from Figure 5 and Figure 6, when there are only four supports, the maximum static deformation of the bed is $0.38 \mu \mathrm{m}$ and the large deformation is concentrated at the positions of the spindle and the tailstock. After adding two supports in succession, the amount of deformation at the position of the spindle and tailstock reduced to less than $0.13 \mu \mathrm{m}$. The maximum deformation is moved to Z3 axis, which has little effect on the polishing accuracy.



(a)

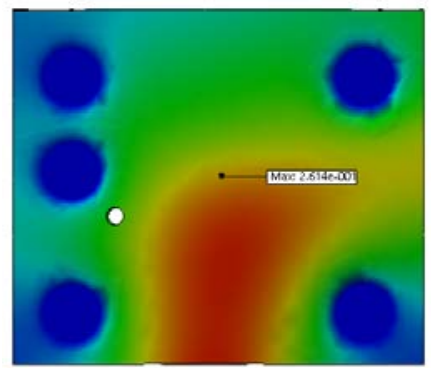

(b)

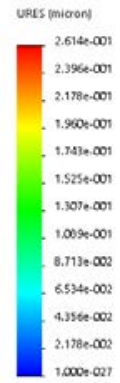

$10000=027$

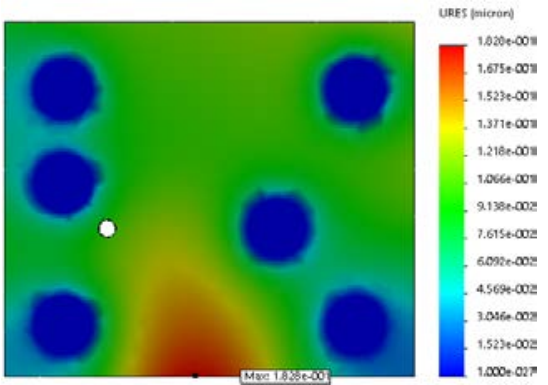

(c)

Fig. 5. Deformation of the granite bed's bottom surface. (a):four supports; (b):five supports; (c):six supports.

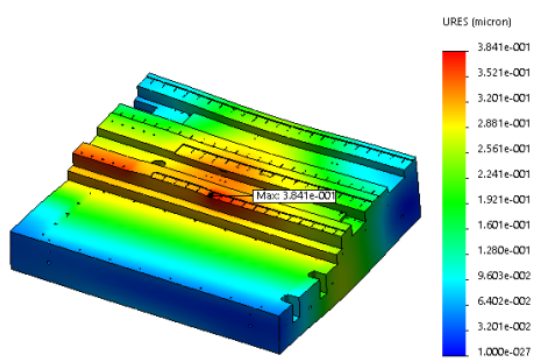

(d)



(e)

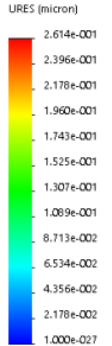

mencos

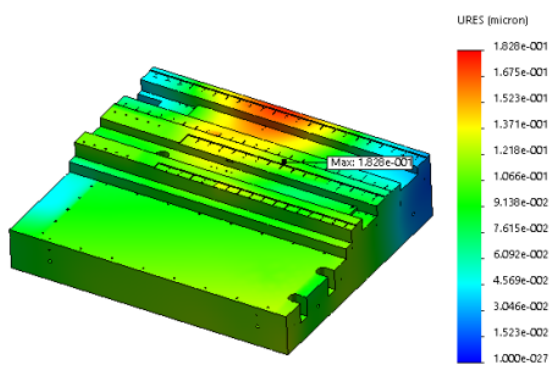

(f)

Fig. 6. Overall deformation on the granite bed. (a): four supports; (b):five supports; (c):six supports.

\subsection{Finite element modal analysis}

In order to prevent the resonance phenomenon of the machine tool in actual operation and affect the stability and reliability of the machining process, we performed a modal analysis on the key components of the machine tool. The possible vibration sources are spindle rotation
$(10 \mathrm{~Hz})$, grinding wheel rotation $(200 \mathrm{~Hz})$ and polishing machine vibration $(10 \mathrm{~Hz})$. The modal analysis results are shown in Table 2:

According to the data in Table 2, the key components of the machine tool will not cause resonance when the machine is running normally.

Table 2. FEA modal analysis of key components.

\begin{tabular}{|c|c|c|c|c|c|}
\hline \multirow{2}{*}{ Modal order } & \multicolumn{5}{|c|}{ FEA calculation frequency/Hz } \\
\cline { 2 - 6 } & Bed & Spindle base & Tailstock base & Polishing module's slide & Turing/Grinding module's slide \\
\hline 1 & 880.33 & 99.863 & 91.943 & 1124.8 & 3045.4 \\
\hline 2 & 1028.9 & 99.979 & 116.29 & 1427.8 & 3333.4 \\
\hline 3 & 1110.3 & 241.13 & 189.44 & 1821.8 & 6127.4 \\
\hline 4 & 1225.8 & 366.15 & 333.2 & 3682.9 & 9360.9 \\
\hline 5 & 1304.4 & 459.91 & 337.23 & 4347.8 & 9583.6 \\
\hline
\end{tabular}

\subsection{Simulation of processing conditions}

In order to verify whether the model has motion interference in actual processing, we assembled the workpiece with the machine tool for kinematic analysis, and the actual processing space relationship is shown in
Figure 7. After kinematics simulation, it is verified that the structure and stroke of the machine tool can complete the machining of the expected shaft parts, as well as the aspheric turning of some optical parts. 


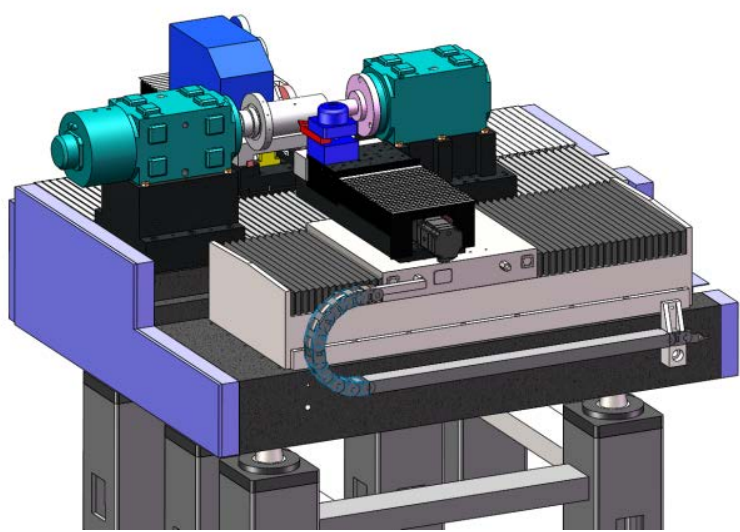

(a)

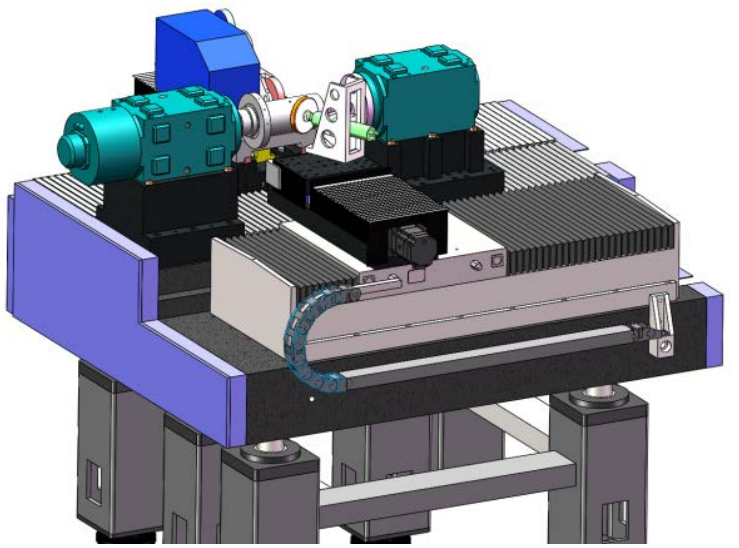

(b)



(c)

Fig. 7. Machining kinematics simulation. (a): $\Phi 150 \mathrm{~mm}$ shaft's turning; (b): $\Phi 150 \mathrm{~mm}$ shaft's grinding; (c): $\Phi 500 \mathrm{~mm}$ parabolic mirrors' turning.

\section{Conclusions}

In this paper, a multifunctional composite machine tool with functions of turning, grinding and abrasive belt polishing is designed to meet the machining accuracy requirements of ultra-precision shaft parts. The traditional machining processes of shaft parts are integrated into one device, which improves the automation level of machining while ensuring high precision. The main conclusions are summarized as follows:

1. The feasibility of ultra-precision deterministic figuring of shaft parts was verified by experiments;

2 . It is verified that the deterministic figuring process requires entrance conditions, and the values of the entrance conditions are determined based on simulation and experimental results;

3. Based on the actual requirements, the design requirements of the new machine tool are put forward in terms of function and structure;

4. The structure of the composite machine tool is designed, and the performance parameters of the components are selected according to the machining process, the kinematics simulation of the actual machining was performed by CAD software, the deformation and modal of the machine tool parts were analyzed and optimized by FEA software, which verified the rationality of the machine tool design;

5. According to the motion accuracy and geometric tolerances of each component, it is predicted that after turning and grinding, the typical parts can reach the entrance conditions of $1 \mu \mathrm{m}$ roundness and $3 \mu \mathrm{m}$ cylindricity, which lays the foundation for the abrasive belt polishing to achieve the final precision of $0.1 \mu \mathrm{m}$ roundness and $1 \mu \mathrm{m}$ cylindricity.

\section{Acknowledgments}

This project was supported by the National Natural Science Foundation of China (grant no. 51835013, 51991371), National Key Research and Development Plan of China(No.2016YFB1102304), and National Defense Basic Research Challenges in Nuclear Science. Our deepest gratitude goes to the reviewers for their careful work and thoughtful suggestions that have helped improve this paper substantially. We would like to express our gratitude to professors and students from Hunan Key Laboratory of Ultra-Precision Machining Technology for their helpful participation and engagement. 


\section{References}

1. W.L. Xiong, Chin. J. Mech. Eng. 47(5), 40-58 (2011)

2. G.Q. Zhang, H.C. Yu, Manu. Tech. Mach. Tool, 3, 55-59 (2018)

3. W.H. Zhu, M.B. Jun, Y. Altintas, Int. J. Mach. Tool. Manu. 41(7), 953-965 (2001)

4. Aleksandrova, S. Irina, J. Mech. Eng. 65(2), 87-102 (2019)

5. X.L. Chen, Y.F. Dai, Materials, 12(9), 1389 (2019)

6. C. Liu, R\&D of precision roller lathe CNC system based on UMAC. Guangdong University of Technology (2018)
7. Q. Hu, J.D. Liu, P.N. Zhao, Manu. Tech. Mach. Tool, 12, 66-69 (2019)

8. H.P. Chen, Design and performance analysis of hydrostatic guideway for precision machine. Huazhong University of Science and Technology, (2019)

9. H. Xia, H.L. Cui, Y.H. Liu, Mach. Tool. Hydraulics, 46(4), 84-96 (2018)

10. F. Kang, J.W. Fan, Mech. Sci. Tech. Aerosp. Eng. 27(5), 588-591 (2008) 